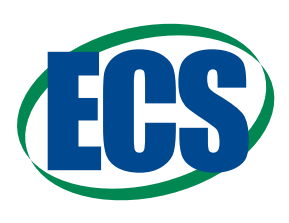

JSS Focus Issue on Nanocarbons-In Memory of Sir Harry Kroto

\title{
Review-Single-Walled Carbon Nanohorn-Based Dye-Sensitized Solar Cells
}

\author{
F. Lodermeyer, R. D. Costa, ${ }^{\mathrm{Z}}$ and D. M. Guldi* ${ }^{*, \mathrm{z}}$ \\ Department of Chemistry and Pharmacy, Friedrich-Alexander-University Erlangen-Nuremberg, 91058 Erlangen, \\ Germany
}

\begin{abstract}
Efforts in the broad field of dye-sensitized solar cells (DSSC) are governed by four key challenges: i) the development of photoelectrodes with high charge injection yields and charge collection efficiencies, ii) the development of low-cost buffer layers, which effectively reduce the back reaction of electrons from the transparent conductive oxide (TCO) to the electrolyte, iii) the design of platinum-free counter electrodes (CE), which feature good electrolyte regeneration yields, and iv) the implementation of (quasi) solid-state and iodine-free electrolytes featuring excellent ionic diffusion and conductivity. The introduction of nanocarbons into each component of DSSCs has emerged as a promising strategy to tackle all the aforementioned challenges. Leading examples of nanocarbons are graphene, carbon black (CB), multi-walled carbon nanotubes (MWCNT), and single-walled carbon nanotubes (SWCNT). Here, we focus on single-walled carbon nanohorns (SWCNH), which were successfully integrated into each of the aforementioned parts of DSSCs.

(C) The Author(s) 2017. Published by ECS. This is an open access article distributed under the terms of the Creative Commons Attribution 4.0 License (CC BY, http://creativecommons.org/licenses/by/4.0/), which permits unrestricted reuse of the work in any medium, provided the original work is properly cited. [DOI: 10.1149/2.0241706jss] All rights reserved.

(cc) BY
\end{abstract}

Manuscript submitted December 28, 2016; revised manuscript received February 8, 2017. Published February 25, 2017. This paper is part of the JSS Focus Issue on Nanocarbons - In Memory of Sir Harry Kroto.

\section{Contemporary Challenges in DSSCs}

The utilization of low to medium purity materials via low-cost processes makes DSSCs very attractive for practical developments. In addition, the modular nature of DSSCs enables easy and flexible fabrication. DSSCs consist of two parallel TCO glass substrates with a high optical transmittance $(>90 \%)$ and a low sheet resistance $(8-$ $15 \Omega / \square){ }^{1,2}$ These substrates are normally coated with a thin layer of fluorine-doped tin oxide (FTO) or indium-doped tin oxide (ITO). On the front electrode, which is the photoanode for n-type DSSCs and the photocathode for p-type DSSCs, a mesoporous nanoparticle-based layer of a wide bandgap semiconductor is deposited. ${ }^{1}$ The thickness ranges from several nanometers to tens of micrometers. By virtue of a slow sintering at high temperatures, a mesoporous structure is realized featuring increased surface area, when compared to a flat architecture. This is crucial in terms of maximum dye adsorption and high charge injection yields. To ensure good contact between the mesoporous electrode and the TCO as well as to reduce recombination from the TCO to the electrolyte, a buffer layer is applied. To this end, a nm-sized $\mathrm{TiO}_{2}$ layer is deposited in, for example, n-type DSSCs, by a $\mathrm{TiCl}_{4}$ treatment. ${ }^{3,4}$ Another key aspect is the complete monolayer coverage of the semiconductor with the dye. It serves as both, a blocking layer against recombination and an absorber for the visible part of the solar spectrum. ${ }^{5}$ The CE consists of a material that effectively recovers the electrolyte species to close the DSSC circuit. In standard DSSCs, a thin platinum-based layer, which transforms the oxidized electrolyte (e.g., $\left.\mathrm{I}_{3}^{-}, \mathrm{Co}^{3+}, \mathrm{Fc}^{+}\right)$to their reduced form (e.g., $\mathrm{I}^{-}, \mathrm{Co}^{2+}$, Fc) species, is used. ${ }^{6,7}$ Typically, low-viscosity solvents are employed for the electrolyte. ${ }^{8,9}$ Given, however, the long-term instability, when organic solvents are used, research focusses on novel solid-state electrolytes with high ionic conductivities (vide infra).

DSSCs have been advertised as a viable alternative for siliconbased solar cells, but their efficiencies still fall short compared to the latter. $^{10}$ To this date, a number of obstacles limit the efficiencies of DSSCs and, as such, hamper their commercialization. Firstly, low charge collection efficiencies at the photoelectrode arise from an inefficient charge transport within the mesoporous semiconductor network due to charge recombination. Secondly, standard $\mathrm{TiCl}_{4}$-based buffer layers hamper industrial manufacturing. On one hand, a fast and irreversible degradation of $\mathrm{TiCl}_{4}$-based solutions under ambient

*Electrochemical Society Member.

${ }^{\text {z}}$ E-mail: ruben.costa@fau.de; dirk.guldi@fau.de conditions and, on the other hand, its acidic nature impose major limitations. Thirdly, the CE is a FTO substrate covered with a platinum layer. Platinum is a rare earth metal, which use increases the production costs of DSSCs. Finally, the use of a liquid electrolyte has both temperature and long-term stability issues. At low temperatures the organic solvent can solidify, while at elevated temperatures it can evaporate.

Given the remarkable features of SWCNHs (vide infra), all of the aforementioned obstacles are tackled upon implementing them into each part of a DSSC. Firstly, the limiting charge transport of pristine semiconductor networks is overcome by SWCNHs, when used as integrative materials to yield highly conductive hybrid photoelectrodes. Secondly, SWCNH-based buffer layers increase the efficiencies of DSSCs and reduce the production of chemical waste. Thirdly, the replacement of platinum by SWCNHs as CE material is successful. Finally, solvent-based electrolytes are replaced by solid-state and quasi solid-state electrolytes based on ionic liquids. Here, SWCNHs assist in increasing the ionic diffusivity to yield high regeneration efficiencies of the electrolyte. Herein, we reviewed all their achievements.

\section{Definition, Properties, and Characterization of SWCNHs}

Linking a one-dimensional graphene sheet, while welding and removing wedges, will eventually result in a three-dimensional, conical shape. The resulting apex is defined by one or more declinations in the form of pentagons. ${ }^{11,12}$ Like graphene as well as SWCNTs and MWCNTs, these new carbon nanocones and nanohorns are defined by a zero Gaussian curvature. ${ }^{12}$ SWCNHs are one class of such carbon nanocones with a defined, sharp apical angle of $20^{\circ}$. Their synthesis encompasses metal-free and high-yield routes based on $\mathrm{CO}_{2}$ laser ablation of graphite. ${ }^{13}$ Besides, multi-walled carbon nanohorns have been isolated as a side-product of arc discharge synthesis of SWCNHs. ${ }^{14}$ Typical diameters range from 2 to $5 \mathrm{~nm}$ with a length distribution from 30 to $50 \mathrm{~nm}$. Hereby, the angle of the conical tip of $20^{\circ}$ is given by the defined curvature of five pentagons at the apex. ${ }^{15,16}$ In solution, they associate into round-shaped and loosely bound aggregates reaching sizes of around $100 \mathrm{~nm}$. Either "dahlia"-shaped or "bud"-like aggregates are formed depending either on the inert gas mixtures based on $\mathrm{He}, \mathrm{Ar}$, and $\mathrm{N}_{2}$ or the corresponding pressures. ${ }^{17,18}$

Opening angles wider than $20^{\circ}$ correspond to fewer pentagonal declinations, while increasing the number of pentagons at the tip results in sharper apical angles. In this review, the focus is, however, placed exclusively on SWCNHs defined by five pentagons near the 
apex. ${ }^{19}$ Highly nonuniform effective local fields for states located at the cone upon applying an external field are a result of the variation in the local sheet orientation near to the tip and the two-dimensional nature of the electronic states. ${ }^{15}$ New Landau levels associated with Lissajous orbits that circle the apex multiple times are generated by external electric or magnetic fields. This three-body scenario generates transport phenomena governed by highly inhomogeneous local fields similar to those created by the defects in graphene sheets. As such, local regions of enhanced reactivity and electron density evolve around the apex with consequences for charge transport and reactivity. This renders SWCNHs as interesting material for solar and energy storage applications. ${ }^{20,21}$

Their large surface area and porosity enable their dispersibility in common organic solvents at concentrations as high as $5 \mathrm{mg} \mathrm{mL}^{-1} .^{21-23}$ SWCNHs can be applied without further purification. In fact, they are produced at scales of around $1 \mathrm{~kg} \mathrm{day}^{-1} \cdot{ }^{24,25}$ Complementary methods comprise pulsed arc discharge, ${ }^{26}$ torch arc in open air, ${ }^{27}$ and cavity arc jet in open air. ${ }^{28}$

Regarding spectroscopy, the Raman spectrum of SWCNHs reveals graphitic and amorphous carbon-like features. ${ }^{16,29}$ Upon exciting at $532 \mathrm{~nm}$, two prominent bands evolve at around 1333 and $1594 \mathrm{~cm}^{-1}$ The latter corresponds to the symmetric $E_{2 g}$ mode at the $\Gamma$ point ( $\mathrm{G}$ mode), whereas the earlier is of $\mathrm{A}_{1 \mathrm{~g}}$ symmetry and is induced by defects or edges (D mode) ${ }^{30}$ The $\mathrm{G}$ band is commonly found is all $\mathrm{sp}^{2}$ carbon systems arising from in-plane stretching of C-C bonds. ${ }^{31}$ The basal plane is disturbed by functionalization or defects introducing localized vibrational modes or increasing disorder. As a result, the $\mathrm{G}$ band is broadened. SWCNHs feature like other $\mathrm{sp}^{2}$-carbon systems a $2 \mathrm{D}$ band around $2655 \mathrm{~cm}^{-1}$. Notably, SWCNHs exhibit a high $\mathrm{I}_{\mathrm{D}} / \mathrm{I}_{\mathrm{G}}$ ratio due to defects often exceeding a value of $1 .{ }^{13,32}$

Concerning their electronic features, the stability, optimum geometry, and electronic properties of SWCNHs are significantly influenced by their unique conical structure. ${ }^{16,19,33}$ Scanning tunneling microscopy and simulated scanning tunneling microscopy assays indicated a net electron transfer toward the pentagon vertex sites. ${ }^{19}$ The local density of states (LDOS) grows rapidly at the tip region. ${ }^{33}$ Here, oxidized SWCNHs (ox-SWCNHs) behave like p-type semiconductors, whereas pristine SWCNHs exhibit n-type semiconductors. ${ }^{34,35}$ Upon applying oxidative conditions to SWCNHs, such as mixtures of $\mathrm{H}_{2} \mathrm{SO}_{4} / \mathrm{H}_{2} \mathrm{O}_{2}$ or $\mathrm{HNO}_{3}$, the pentagons of SWCNHs are annihilated resulting in different electrical conductivity responses. Furthermore, oxidation of SWCNHs increases their porosity and specific surface area by a factor of $\sim 5 .^{36,37}$

Taking their electromagnetic properties into account, electron spin resonance (ESR) and static magnetic susceptibility measurements revealed at least one unpaired electron in individual SWCNHs and a small contribution of diamagnetic susceptibility. The latter lies above fullerenes, but below that of MWCNTs and bulk graphite. ${ }^{38}$ Two components in SWCNH aggregates were distinguished by high-resolution ${ }^{13} \mathrm{C}$ NMR. ${ }^{39}$ Firstly, a fast relaxation component with a chemical shift of $124 \mathrm{ppm}$ is assigned to a carbon nanotube-like character. A fast spin-lattice mechanism is caused by rapid spin diffusion to localized paramagnetic centers arising from structural defects. Secondly, a slow relaxation component with a chemical shift of $116 \mathrm{ppm}$ corresponds to the graphite-like core structure.

The good dispersibility of SWCNHs allows an easy preparation of thin films by solvent-based techniques. ${ }^{40} \mathrm{X}$-ray diffraction analyses revealed that SWCNH-based thin films exhibited van der Waals distances of $0.4 \mathrm{~nm}$ compared to $0.336 \mathrm{~nm}$ found in ordinary graphite. Therefore, SWCNHs feature micro- and mesopores due to their specific aggregate structure. ${ }^{16}$

Given their specific morphology and their catalytic behavior, SWCNH-based films were used for biomedical applications, ${ }^{41-46}$ gas storage and detection applications,${ }^{47-50}$ fuel cell applications, ${ }^{51-55}$ and reinforcement nanocomposites. ${ }^{56,57}$ Here, the application of SWCNHs into DSSCs as integrative component of the photoelectrode, buffer layers, platinum-free CEs, and additives for (quasi) solid-state electrolytes is discussed.

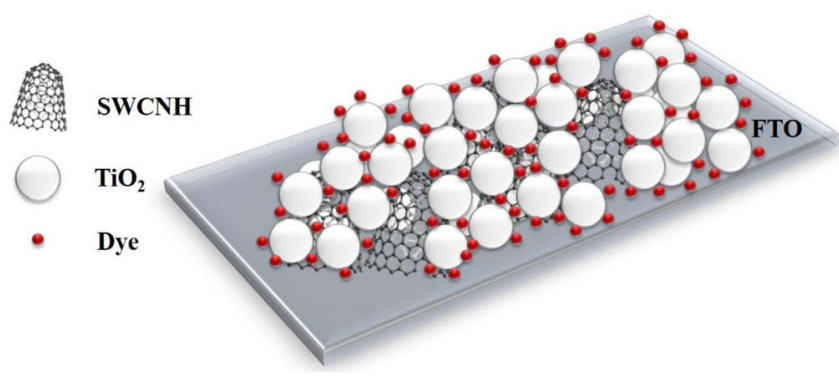

Figure 1. Schematic representation of a $\mathrm{SWCNH} / \mathrm{TiO}_{2}$ hybrid photoelectrode.

\section{SWCNH-Based Hybrid Photoelectrodes}

A key aspect for highly efficient DSSCs is the optimization of the charge transport across the mesoporous semiconductor network. Upon injection, electrons are prone to various trapping and detrapping events along grain boundaries as they are present in the photoelectrodes. ${ }^{58,59}$ In other words, the electron-hole recombination rate has to be low for high power conversion efficiencies. ${ }^{60,61}$ In this regard, the fraction between charge transport resistance $(R w)$ and the resistance toward recombination $\left(R_{k}\right)$ defines the charge collection efficiency $\left(\eta_{c c}\right)$ according to Equation 1.59

$$
\eta_{c c}=1-R_{w} / R_{k}
$$

$R w$ is the electron transport resistance under illumination conditions and $R_{k}$ is the resistance toward recombination under dark conditions applied in electrochemical impedance spectroscopy (EIS) assays.

The integration of nanocarbons into the semiconductor network has emerged as a viable strategy to enhance the $\eta_{c c}$ s of photoelectrodes. They serve as electron acceptors and assist the ballistic charge carrier transport for effective charge separation and rapid transport of charge carriers in nanocarbon / $\mathrm{TiO}_{2}$ hybrid photoelectrodes. ${ }^{62-64}$ Prominent examples of nanocarbons in $\mathrm{TiO}_{2}$ hybrid photoelectrodes are MWCNTs, SWCNTs and graphene. ${ }^{62,63,65-68}$ The implementation of pristine nanocarbons typically is impeded by their tendency to bundle in solution and their poor dispersability. ${ }^{23}$ Owing to their good dispersibility in common solvents (vide supra), SWCNHs were easily applicable in $\mathrm{TiO}_{2}$-based photoanodes. ${ }^{65}$

SWCNH / $\mathrm{TiO}_{2}$ hybrid composites were prepared by drop-wise addition of SWCNH dispersions to yield weight percentages of 0.1 , $0.2,0.5$, and $0.7 \mathrm{wt} \%$ (Figure 1). The resulting pastes were stirred prior to their use to ensure homogenous mixing of all components, doctor bladed onto FTO substrates, and sintered at $400{ }^{\circ} \mathrm{C}$ for 30 min. Notably, inside the $\mathrm{TiO}_{2}$ network the SWCNHs do not degrade upon sintering. Raman signals at 1275 and $1595 \mathrm{~cm}^{-1}$ further supported these findings. They were assigned to the $\mathrm{D}$ and $\mathrm{G}$ band of the SWCNHs present in the $\mathrm{TiO}_{2}$ network, respectively. Furthermore, signals at 397,517 , and $639 \mathrm{~cm}^{-1}$ were ascribed to the $B_{1 g}, A_{1 g}+B_{2 g}$, and $\mathrm{E}_{\mathrm{g}}$ modes in $\mathrm{TiO}_{2}$ anatase nanoparticles, respectively. ${ }^{69^{\circ}} \mathrm{X}$-ray diffraction (XRD) measurements confirmed that the $\mathrm{TiO}_{2}$ pattern was not affected by the implementation of SWCNHs. Scanning electron microscopy (SEM) measurements, desorption experiments, diffuse reflectance, and $\alpha$-step profilometry measurements gave insights into the microstructure of the $\mathrm{SWCNH} / \mathrm{TiO}_{2}$ hybrid films. The roughness and, as a consequence, the amount of adsorbed dye and light-scattering increased upon implementing SWCNHs. As a matter of fact, the aforementioned effects contributed to an increase in short-circuit current densities $\left(J_{s c}\right)$ compared to that of the reference device without SWCNHs. Additionally, the work functions measured by the Kelvin probe technique increased upon SWCNH addition in good agreement with the literature. ${ }^{70,71}$ Accordingly, the $\mathrm{TiO}_{2}$ film resistances reduced from $\sim 800$ to $\sim 100 \mathrm{M} \Omega$ for pristine $\mathrm{TiO}_{2}$ and $0.5 \mathrm{wt} \%$ SWCNH-containing electrodes, respectively. 
To gain insights into the electron conducting properties, transient absorption measurements were performed. Upon exciting at $387 \mathrm{~nm}$, the bleaching recovery at $460 \mathrm{~nm}$ of the photoexcited dye (N719) was observed. The derived lifetimes reflect the charge recombination and, in turn, electron injection into the SWCNHs. As a matter of fact, the longest lifetimes of $208 \mathrm{~ns}$ were detected for $0.5 \mathrm{wt} \% \mathrm{SWCNH}$ containing $\mathrm{TiO}_{2}$ hybrid films. The efficiencies of reference DSSCs with $4.5 \%$ increased to $8.0 \%$ for the $0.5 \mathrm{wt} \% \mathrm{SWCNH}$-containing DSSCs. In this context, due to improved charge collection efficiencies, $J_{s c} \mathrm{~s}$ and fill factors $(F F \mathrm{~s})$ improved from 10.9 to $16.1 \mathrm{~mA} \mathrm{~cm}^{-2}$ and 0.57 to 0.67 , respectively. $\eta_{c c}$ s rose from $58.7 \%$ to $74.4 \%$ for reference and $0.5 \mathrm{wt} \%$ SWCNH-containing DSSCs, respectively.

\section{SWCNH-based Buffer Layer}

Recombination processes at the interface between TCO and the electrolyte dominate in the absence of a buffer layer. ${ }^{72,73}$ As such, a low shunt resistance of the solar cell reduces the final efficiencies. In $\mathrm{TiO}_{2}$-based DSSCs, a nm-sized layer of $\mathrm{TiO}_{2}$ is deposited onto TCO via hydrolyzing aqueous solutions of $\mathrm{TiCl}_{4}$. This prevents the shunting of charge carriers from the TCO and, in turn, increases the $F F$ s. $^{1}$ With a buffer layer, a higher charge carrier concentration in the semiconducting network evolves accounting for an increase in the quasi-Fermi level. ${ }^{74}$ As a consequence, open-circuit voltages $\left(V_{o c}\right)$ rise (Equation 2). ${ }^{75,76}$

$$
V_{o c}=\left(k_{B} \cdot T / e\right) \cdot \ln \left(I_{i n j} / k_{r e g} \cdot n_{C B} \cdot\left[I_{3}^{-}\right]\right)
$$

where $k_{B}$ is the Boltzmann constant, $T$ is the absolute temperature, $e$ is the elementary charge, $I_{\text {inj }}$ is the flux of injected electrons from the sensitizer, $n_{C B}$ is the charge density in the conduction band, $k_{\text {reg }}$ is the electrolyte regeneration rate constant, and $\left[\mathrm{I}_{3}{ }^{-}\right]$is the concentration of $\mathrm{I}_{3}{ }^{-}$in the bulk electrolyte.

Smoothening of the rough TCO surface by a buffer layer is another effect and assists in realizing higher photovoltages: ${ }^{77-79}$ the dark current is effectively suppressed and the charge carrier lifetime $\left(\tau_{n}\right)$ increases. ${ }^{1,80}$ The latter is studied via EIS, intensity-modulated photovoltage (IMVS), or open-circuit voltage decay (OCVD).$^{59,80-82}$ The OCVD allows a continuous monitoring of $\tau_{n}$ as a function of $V_{o c}$ (Equation 3):

$$
\tau_{n}=-k_{B} \cdot T / e \cdot\left(d V_{o c} / d t\right)^{-1}
$$

In other words, $\tau_{n}$ as a function of $V_{o c}$ is defined by the reciprocal of the derivative of the $V_{o c}$ decay normalized by the thermal voltage. With this approach, $n_{\mathrm{CB}}$ is determined, since the photovoltage is maintained by the residual charge carriers present in the photoelectrode. The trapped electron density outnumbers the free electron density by several orders of magnitude, since $\mathrm{TiO}_{2}$-based DSSCs possess a large number of trap states (vide supra). Therefore, OCVD is best described as the average of free charge carrier lifetime in dependence of the balance of trapped and free charge carriers. ${ }^{80}$ For identical photoelectrodes, in which several buffer layers are employed, OCVD represents a powerful tool to study electrode recombination process at the TCO|electrolyte surface.

Uon applying a buffer layer, the charge carrier diffusion length $\left(L_{\text {eff }}\right)$ increases (Equation 4)..$^{59}$

$$
L_{e f f}=\left(D_{e f f} \cdot \tau_{n}\right)^{1 / 2}
$$

$D_{\text {eff }}$ is the effective electron diffusion coefficient calculated using Equation 5:

$$
D_{e f f}=R_{k} / R_{w} \cdot d^{2} \cdot k_{e f f}
$$

where $k_{\text {eff }}$ is the reaction rate constant for the recombination of the electrons at the TCO with the oxidized form of the redox couple defined by the minimum of the semiconductor semicircle in EIS measurements and $d$ the electrode thickness.

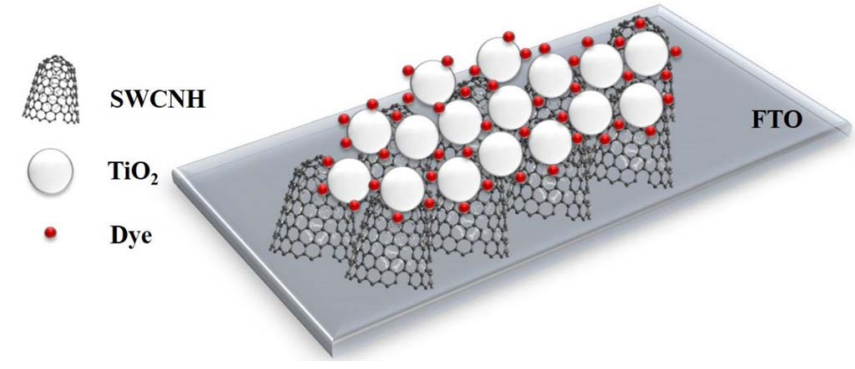

Figure 2. Schematic representation of a SWCNH-based buffer layer.

The advantage of using nanocarbon-based buffer layers is that their work function lies well below the conduction band edge of $\mathrm{TiO}_{2}{ }^{83}$ For example, a dispersion of reduced graphene oxide (rGO) was spin coated onto TCO substrates to yield $500 \mathrm{~nm}$ buffer layers. ${ }^{83} V_{o c} \mathrm{~s}$ and $F F$ s increased due to a reduced electron recombination processes, which was further corroborated in EIS measurements. The efficiencies improved from $4.9 \%$ to $5.3 \%$. However, graphene oxide (GO) was used as starting material for good dispersibility and mixing with $\mathrm{TiO}_{2}$. This required, however, the reduction of GO to yield good conductivity. In contrast, SWCNH dispersions are readily useable to produce buffer layers by means of spin coating (Figure 2). ${ }^{22}$

Buffer layer thicknesses of 5, 15, 20, and $30 \mathrm{~nm}$ were realized by varying the spin velocity and the amount of spin coated SWCNH dispersions $\left(3.6 \mathrm{mg} \mathrm{mL}^{-1}\right.$ in ortho-dichlorobenzene). ${ }^{22}$ The resulting SWCNH-based buffer layers exhibited transmittances close to $98 \%$. To get insights into the film morphology, scanning electron microscopy (SEM) was performed. All FTO substrates were homogenously covered by a thin layer of SWCNHs leaving no bare FTO sites free. This reduced the electron recombination processes between FTO and the electrolyte. Upon increasing the layer thickness, the average roughness of the buffer layer increased resulting in mesoporous-like layers. As a consequence, the contact between the buffer layer and the $\mathrm{TiO}_{2}$ nanoparticles enhanced the connection at the $\mathrm{FTO} \mid \mathrm{TiO}_{2}$ interface. Notably, the average sheet resistance measured by four-point probe of the SWCNH-covered FTO substrates increased from 15.3 to $21.6,24.6$, and $34.1 \mathrm{k} \Omega$ for buffer layer thicknesses of 5, 15, 20, and $30 \mathrm{~nm}$, respectively. In this regard, 5 and $15 \mathrm{~nm}$ thick buffer layers exhibited resistances similar to that of $\mathrm{TiCl}_{4}$-treated reference substrates with an average sheet resistance of $18.0 \mathrm{k} \Omega$.

Next, SWCNH-based buffer layers were tested $\mathrm{TiO}_{2}$-based DSSCs with N719 as photosensitizer and $\mathrm{I}^{-} / \mathrm{I}_{3}{ }^{-}$as electrolyte. When comparing SWCNH-based buffer layers versus non-treated FTO substrates, an increase of $J_{s c} \mathrm{~s}, V_{o c} \mathrm{~s}, F F \mathrm{~s}$, and efficiencies was observed: $J_{s c} \mathrm{~s}$, $V_{o c} \mathrm{~s}$ and $F F$ s increased from 9.4 to $16.4 \mathrm{~mA} \mathrm{~cm}^{-2}, 0.76$ to $0.78 \mathrm{~V}$, and 0.60 to 0.64 for non-treated and $15 \mathrm{~nm}$ thick SWCNH-based buffer layer, respectively. Consequently, the corresponding efficiencies almost doubled from $4.3 \%$ to $8.2 \%$. Incident photon-to-current conversion efficiency (IPCE) measurements further confirmed the increased $J_{s c} \mathrm{~s}$. Here, devices with $15 \mathrm{~nm}$ thick buffer layers resulted in maximum IPCEs of $80 \%$. The latter dropped to $50 \%$ and to $40 \%$ for DSSCs with $30 \mathrm{~nm}$ thick layers and non-treated reference devices, respectively. IPCEs are directly related to light-harvesting efficiencies (LHEs), charge carrier injection efficiency $\left(\eta_{i n j}\right)$, yield of the electrolyte regeneration $\left(\eta_{r e g}\right)$, and $\eta_{c c} s . \eta_{i n j}$ and $\eta_{r e g}$ are close to unity for N719-based electrodes with a liquid $\mathrm{I}^{-} / \mathrm{I}_{3}{ }^{-}$-based electrolyte and only minor impact of the SWCNH-based buffer layers on the LHEs (Equation 6). ${ }^{84}$

$$
I P C E=L H E \cdot \eta_{i n j} \cdot \eta_{r e g} \cdot \eta_{c c}
$$

Therefore, the key aspect of the SWCNH-based buffer layers is the enhancement of $\eta_{c c} \mathrm{~s} .{ }^{75,85}$ Importantly, $\eta_{c c} \mathrm{~s}$ are closely related to $F F \mathrm{~s}$, which are impacted by electron loss processes as well as series and shunt resistances (vide supra).

EIS was applied to gain insights into the underlying device mechanisms. Importantly, $R_{w} \mathrm{~s}$ were strongly influenced by the thickness of 
the SWCNH-based buffer layer. Upon increasing the SWCNH layer thickness from 0 to $5 \mathrm{~nm}, R_{w}$ s reduced from 19.2 to $13.0 \Omega$. Further increasing the thickness from 15 to 20 , to 25 , and to $30 \mathrm{~nm}$ increased, however, $R_{w}$ s from 14.5 to 14.9 , to 17.3 , and to $34.1 \Omega$, respectively. This was ascribed to the increase in the sheet resistance decreasing $\eta_{c c}$ s. $R_{k}$ s increased from 36.3 to 59.1 and to $75.3 \Omega$ for $S W C N H$-based buffer layer thicknesses of 0,5 , and $15 \mathrm{~nm}$, respectively. A further increase in the buffer layer thickness reduced $R_{k}$ s from 74.5 to 70.2 and to $68.9 \Omega$ for DSSCs with layer thicknesses of 20,25 , and $30 \mathrm{~nm}$, respectively. As a consequence, $\eta_{c c}$ s increased from $47.1 \%$ to $78.0 \%$ and to $80.7 \%$ for devices with SWCNH-based buffer layers of 0,5 , and 15 $\mathrm{nm}$, respectively, and decreased from $80.0 \%$ to $75.4 \%$ and to $50.5 \%$ for 20,25 , and $30 \mathrm{~nm}$, respectively. Upon applying SWCNH-based buffer layers, $\tau_{n}$ increased from 3.0 to $4.6 \mathrm{~ms}$ for devices with layer thicknesses of 0 and $5 \mathrm{~nm}$, respectively. $\tau_{n}$ remained constant with 4.6 $\mathrm{ms}$ upon further increasing the layer thickness, but decreased to 3.6 $\mathrm{ms}$, when a layer thickness of $30 \mathrm{~nm}$ was reached. As a consequence, maximum $L_{e f f}$ s of $5.8 \mu \mathrm{m}$ were achieved for DSSCs with a $15 \mathrm{~nm}$ thick buffer layer (Equation 4) resulting in the highest efficiencies of $8.2 \%$. In contrast, $L_{e f f} \mathrm{~s}$ of $3.8 \mu \mathrm{m}$ and efficiencies of $4.3 \%$ were noted for reference devices. As a consequence, SWCNH-based buffer layers represent a viable alternative to the corrosive and hard to recycle $\mathrm{TiCl}_{4}$ treatment.

\section{SWCNH-based Counter Electrodes}

Platinum is widely used as CE material in combination with iodinebased electrolytes. ${ }^{8,86}$ Platinum-based CEs feature low charge transfer resistances $\left(R_{c t}\right)$. Additionally, high limiting diffusion current densities $\left(J_{\text {lim }}\right)$ and large exchange current densities $\left(J_{0}\right)$ are observed from the Tafel polarization curves. $J_{0}$ represents the slope of the Tafel curve and is inversely proportional to $R_{c t}$ (Equation 7). ${ }^{87}$

$$
J_{O}=R \cdot T / n \cdot F \cdot R_{c t}
$$

$R$ is the Avogadro constant, $n$ is the number of electrons transferred in the redox reaction, and $F$ is the Faraday constant.

However, platinum is one of the scarcest elements constituting a major bottleneck for the introduction of DSSCs into the market. ${ }^{2,88-91}$ In addition, platinum catalyzes inefficiently redox couples based on cobalt(II / III) complexes, disulfides / thiolates, and polysulfides. ${ }^{92}$ In this regard, transition metal complexes (TMC) based on earthabundant elements have been widely used to replace platinum..$^{92}$ For example, highly ordered TiN nanotube arrays on metallic Ti foil substrates resulted in efficiencies of $7.7 \%$. The similarity of the electronic structure compared to noble metals and the high electrical conductivity of $\sim 10^{2} \Omega^{-1} \mathrm{~m}^{-1}$ account for a good electrolyte regeneration. ${ }^{93}$ Zhou et al. proposed a solvothermal approach to prepare oxygen-vacancy rich $\mathrm{WO}_{2.72}$ improving the device efficiency from $4.6 \%$ to $8.0 \%$, when compared to $\mathrm{WO}_{3}$, which has significantly less vacancies. ${ }^{94}$ The efficiency of DSSCs with $\mathrm{WO}_{2.72}$-based CEs was comparable to platinum-based CEs. The authors suggested that vacancies and also the increased surface area are responsible for the higher performance of $\mathrm{WO}_{2.72}$.

Conductive polymers, such as poly(3,4-ethyl-enedioxythiophene) (PEDOT), poly (3,4-propylenedioxy-thiophene) (PProDOT), polypyrrole (PPy), and polyaniline (PANI), also exhibit good catalytic activities, low-temperature processability, and good transparency. ${ }^{95}$ Here, PANI-based CEs stand out due to their excellent catalytic activity and conductivity allowing the production of thin and highly transparent films. For example, oriented PANI nanowire arrays have been grown in situ on a TCO substrate featuring superior electrocatalytic performance relative to random networks of as-grown PANI-based CEs. ${ }^{96}$ For the oriented PANI-based CEs, low $R_{c t}$ s of $\sim 1 \Omega$ were noted indicating a good catalytic activity toward the electrolyte reduction. However, the straightforward preparation of PANI-based CEs is difficult.

Given their high specific surface area, superior electrical conductivity, and good catalytic activity toward the reduction of most

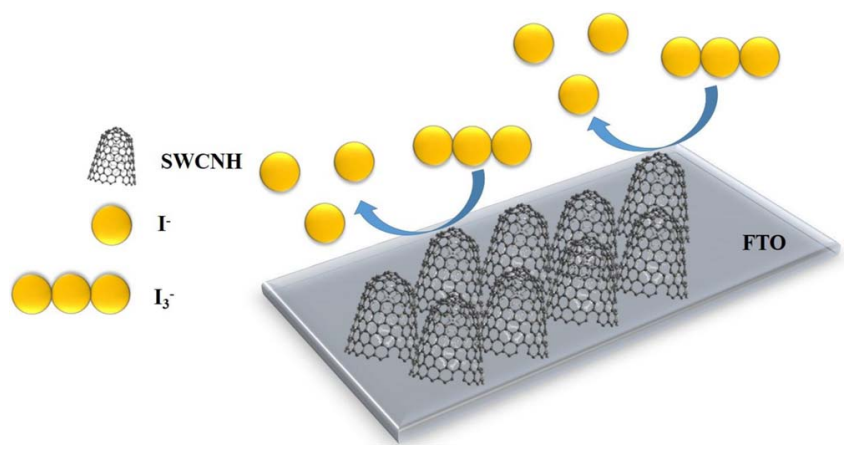

Figure 3. Schematic representation of a SWCNH-based counter electrode.

of the redox couples, nanocarbon-based CEs represent a viable approach to replace platinum. Graphene derivatives, MWCNTs, and CB were already successfully used as highly efficient nanocarbon-based CEs. ${ }^{2,97-104}$

Here, the use of SWCNHs is discussed, as they disperse easily in common organic solvents and feature a high $\mathrm{I}_{\mathrm{D}} / \mathrm{I}_{\mathrm{G}}$ ratio, i.e. high catalytic activity. Firstly, SWCNH-based CEs with thicknesses of 1.5 (CE1), 3.4 (CE2), 6.3 (CE3), and 8.9 (CE4) $\mu \mathrm{m}$ were assembled (Figure 3). ${ }^{23}$ Tafel polarization measurements of symmetric devices with the configuration FTO|SWCNH|electrolyte|SWCNH|FTO revealed information about the interfacial charge transfer properties. Upon increasing the layer thickness, semilogarithmic Tafel plots revealed an increase of $J_{\text {lim }}$ s from 67.0 to 69.0 , to 79.8 , and to 91.6 $\mathrm{mA} \mathrm{cm}{ }^{-2}$ for CE1, CE2, CE3, and CE4, respectively. Similarly, the corresponding $J_{0} \mathrm{~s}$ increased from 27.3 to 32.1 , to 41.6 , and to 42.9 $\mathrm{mA} \mathrm{cm}{ }^{-2}$. As such, thicker SWCNH layers resulted in a higher catalytic activity. EIS measurements of the symmetric cells at $0 \mathrm{~V}$ applied bias gave insights into $R_{c t} \mathrm{~s}$ and Warburg diffusion resistances $\left(R_{\text {diff }}\right)$. From the latter, the diffusion coefficients in the bulk electrolyte $\left(D_{\text {diff }}\right)$ were calculated. ${ }^{59,105}$ SWCNH-based devices featured $R_{c t}$ s below $3 \Omega$, while platinum-based reference CEs featured higher $R_{c t} \mathrm{~s}$ of 5.0 $\Omega$. This indicated a lower catalytic activity toward the electrolyte regeneration of platinum compared to SWCNHs. $D_{\text {diff }}$ increased from 1.1 to 2.2 , to 4.5 , and to $9.1 \times 10^{-10} \mathrm{~m}^{2} \mathrm{~s}^{-1}$ for CE1, CE2, CE3, and CE4, respectively. In contrast, $D_{\text {diff }}$ s of platinum-based CEs with $86.6 \times 10^{-10} \mathrm{~m}^{2} \mathrm{~s}^{-1}$ were significantly higher. This was ascribed to the limited diffusion of the $\mathrm{I}_{3}{ }^{-}$ions inside the mesoporous $\mathrm{SWCNH}$ network.

Finally, $\mathrm{TiO}_{2}$-based DSSCs sensitized with $\mathbf{N 7 1 9}$ and $\mathrm{I}^{-} / \mathrm{I}_{3}{ }^{-}$electrolyte with CE1, CE2, CE3, and CE4 were assembled. $J_{s c}$ and $F F$ s rose upon increasing the layer thickness from 14.2 to 15.1 , to 16.1 , and to $14.4 \mathrm{~mA} \mathrm{~cm}^{-2}$ and $0.47,0.56,0.61$, and 0.61 for DSSCs with CE1, CE2, CE3, and CE4, respectively. The corresponding efficiencies increased from $5.2 \%$ to $6.4 \%$ and to $7.7 \%$ and, finally, decreased to 6.6\%. Notably, DSSCs with CE3 exhibited similar efficiencies compared to platinum-based CEs with $7.9 \%$. From EIS measurements, $R_{c t}$ s of SWCNH-based CEs were around $1 \Omega$, which was significantly lower than those of platinum-based CEs of $7.3 \Omega . D_{\text {diff }}$ s increased from 0.8 to 4.0 and to $8.3 \times 10^{-10} \mathrm{~m}^{2} \mathrm{~s}^{-1}$ for DSSCs with CE1, CE2, and CE3, respectively, and decreased to $6.2 \mathrm{~m}^{2} \mathrm{~s}^{-1}$ for devices with CE4. The diffusion of $\mathrm{I}_{3}{ }^{-}$in the bulk electrolyte was significantly impeded for SWCNH layer thicknesses $\sim 8 \mu \mathrm{m}$ slowing down the dye regeneration. As such, $J_{s c}$ s of DSSCs with CE4 are lower than those of CE3. In contrast, $\mathrm{I}_{3}{ }^{-}$diffusion in reference DSSCs with platinum-based CEs was not impeded due to the nm-sized platinum layer resulting in $D_{\text {diff }} \mathrm{s}$ of $17.3 \times 10^{-10} \mathrm{~m}^{2} \mathrm{~s}^{-1}$. Importantly, DSSCs with CE3 and CE4 both exhibited $F F$ s of 0.61 indicating similar electrolyte regeneration yields. This is further corroborated by similar $J_{0} \mathrm{~s}$ of $41.6 \mathrm{~mA} \mathrm{~cm}^{-2}$ for devices with CE3 and $42.9 \mathrm{~mA} \mathrm{~cm}^{-2}$ for devices with CE4. In summary, SWCNH-based CEs were assembled via a facile and fast drop cast technique without the necessity of a sintering process. SWCNH- 
based CEs feature considerably lower $R_{c t} \mathrm{~s}$ than platinum-based CEs resulting in similar efficiencies.

In an alternative approach, Susmitha et al. used SWCNHs with a $\mathrm{I}_{\mathrm{D}} / \mathrm{I}_{\mathrm{G}}$ ratio of 0.86 produced via arc discharge. ${ }^{106}$ Aqueous dispersions were spray-coated onto FTO substrates and heated to $150^{\circ} \mathrm{C}$. The authors compared the performance of SWCNH-based with graphene nanoplatelets-based (GNP) CEs for DSSCs. Here, SWCNH-based CEs featured efficiencies of $4.1 \%$ with a SWCNH loading of $3.0 \mathrm{mg}$. These are higher than GNP-based CE devices showing efficiencies of $3.1 \%$ with $3.0 \mathrm{mg}$ GNP loading. The main reason for this finding is a higher surface area and a higher amount of catalytically active sites for SWCNH-based CEs. The efficiencies decreased from $4.1 \%$ to $3.7 \%$ for SWCNH higher loadings of $4.0 \mathrm{mg}$. This underlines the importance of the balance between surface area and electrolyte diffusion. Overall, the efficiencies are lower for DSSCs with spraycoated SWCNH-based CEs compared to drop-casted ones probably arising from lower thickness, different morphology, or lower $I_{D} / I_{G}$ ratios. $^{23}$

Considering that the catalytic activity for cobalt-based electrolytes of nanocarbon-based CEs is typically higher compared to platinumbased CEs, DSSCs with SWCNH-based CEs were tested with redox couple based on cobalt complexes. ${ }^{92,107}$ Cobalt-based electrolytes generally exhibit higher $V_{o c}$ s than those of devices with iodide-based electrolytes. ${ }^{8,108,109}$ Carli et al. produced, for example, ox-SWCNHs for a better adhesion to FTO. The $\mathrm{I}_{\mathrm{D}} / \mathrm{I}_{\mathrm{G}}$ ratio increased from $\sim 0.9$ to 1.22 for pristine and ox-SWCNHs, respectively. ${ }^{25}$ ox-SWCNH dispersions in ethanol were spray-coated under a $\mathrm{N}_{2}$ stream onto FTOs and subsequently sintered at $400^{\circ} \mathrm{C}$, while PEDOT- and platinumbased CEs were used as reference devices. From symmetric cells, similar $J_{\text {lim }} \mathrm{s}$ of $\sim 21.0 \mathrm{~mA} \mathrm{~cm}{ }^{-2}$ were observed for SWCNH- and oxSWCNH-based CEs. At the same time, PEDOT- and platinum-based CEs exhibited higher $J_{\lim } \mathrm{s}$ of 22.7 and $25.4 \mathrm{~mA} \mathrm{~cm}^{-2}$, respectively. EIS and J-V measurements of DSSCs with SWCNH-, ox-SWCNH-, and PEDOT-based CEs revealed $R_{c t} \mathrm{~s}$ of $0.21,0.75$, and $0.18 \Omega$ and efficiencies of $6.8 \%, 6.8 \%$, and $6.7 \%$, respectively.

\section{SWCNH-based (quasi) Solid-state Electrolytes}

For highly efficient DSSCs, the regeneration yields of both the oxidized dye and the oxidized species of the electrolyte have to be close to unity to achieve high $J_{s c} \mathrm{~s}$ and $F F \mathrm{~s}$. Additionally, $D_{\text {diff }} \mathrm{s}$ have to be high for a good regeneration of the oxidized dye. ${ }^{110,111} D_{\text {diff }} \mathrm{s}$ are indirectly proportional to $R_{\text {diff }} \mathrm{s}$ according to Equation $8 .{ }^{59}$

$$
D_{\text {diff }}=k_{B} \cdot T / n^{2} \cdot e^{2} \cdot R \cdot c \cdot R_{\text {diff }} \cdot \delta
$$

Iodine-based electrolytes suffer severe limitations due to their prominent absorption features, their corrosive nature, and pronounced charge recombination processes. The long-term stability is especially critical for DSSCs using liquid-based electrolytes due to $\mathrm{I}_{3}{ }^{-}$-induced corrosion at the electrode contacts, on one hand, and solvent solidification and evaporation at low and elevated temperatures, on the other hand. ${ }^{112,113}$ Therefore, novel approaches for preparing iodine-free and solid-state electrolytes featuring excellent ionic diffusivity and thermal stability are highly desired for highly efficient and long-term stable solid-state DSSCs (ssDSSC).

Firstly, the design of novel photoelectrodes allowing for an efficient electrolyte penetration into the mesopores along with a simultaneous enhancement of the light-scattering events is currently under investigation. ${ }^{114-117}$ For example, Ahn et al. synthesized novel honeycomb-like organized $\mathrm{TiO}_{2}$-based photoanodes as a means to increase the dye loading, light-scattering, and electrolyte wetting properties. ${ }^{115}$ The graft copolymer poly(vinyl chloride)-gpoly(oxyethylene methacrylate) (PVC-g-POEM) and $\mathrm{TiO}_{2}$ nanoparticles were directly mixed in a one-step synthesis forming selfassembled and hydrophilic $\mathrm{TiO}_{2}$ nanocrystals. Honeycomb-like structures were generated with PVC-g-POEM / $\mathrm{TiO}_{2}$ hybrid pastes resulting in efficiencies of $7.4 \%$ with a polymer-based electrolyte.

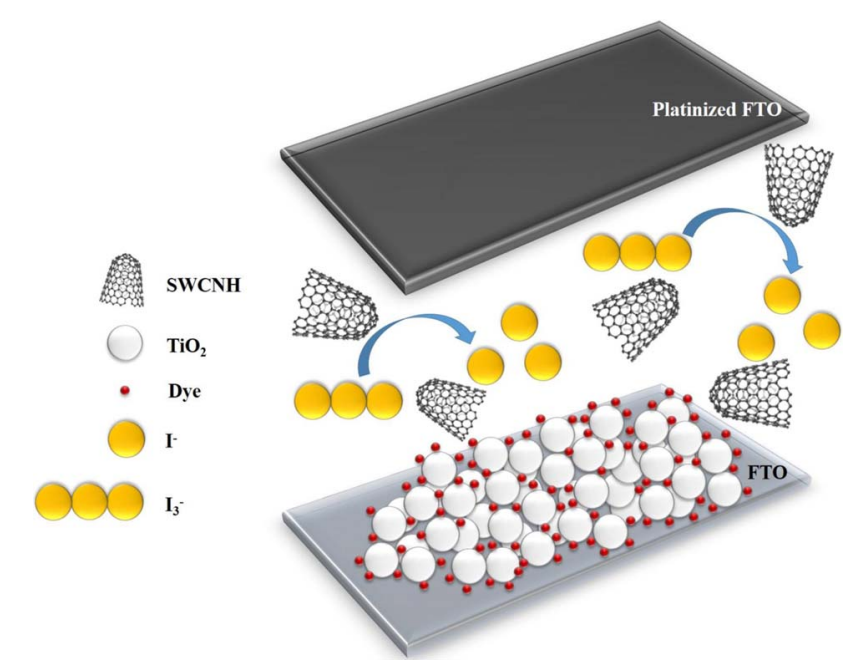

Figure 4. Schematic representation of the integration of SWCNHs into the electrolyte.

Secondly, hole transport materials (HTMs) ${ }^{118}$ and conductive polymers ${ }^{114}$ represent viable alternatives to typical iodide-based electrolytes. Starting with the pioneering work of Bach et al. in 1998 , efficiencies of $\sim 6 \%$ have been achieved with $\mathrm{N}^{2}, \mathrm{~N}^{2}, \mathrm{~N}^{2^{\prime}}, \mathrm{N}^{2^{\prime}}$, $\mathrm{N}^{7}, \mathrm{~N}^{7}, \mathrm{~N}^{7^{\prime}}, \mathrm{N}^{7^{\prime}}$-octakis(4-methoxyphenyl)-9, $9^{\prime}$-spirobi[9H-fluorene]-' $2,2^{\prime}, 7,7^{\prime}$-tetramine (Spiro-MeOTAD) as HTM in ssDSSCs. ${ }^{118}$ Benchmark efficiencies of HTM-based ssDSSCs are today limited up to $\sim 7.7 \%$. Recently, alternative HTMs, such as copper phenantroline have provided efficiencies with $8.2 \% .^{119,120}$

Finally, the integration of nanocarbons, such as $\mathrm{CB},{ }^{121,122} \mathrm{SWC}$ NTs as well as MWCNTs, ${ }^{123,124}$ and graphene ${ }^{125}$ as additives to iodinefree and solid-state electrolytes constitutes a viable strategy to realize ssDSSCs. ${ }^{21,126}$ The benefits of implementing nanocarbons into solidstate electrolytes stand out given their significant improvement of the ionic diffusivity, their catalytic activity toward the reduction of the oxidized form of the electrolyte, and the reduction of the internal resistance of the bulk electrolyte. ${ }^{32,122,127}$

1-ethyl-3-methylimidazolium iodide ([EMIM]I) was mixed with different weight ratios of SWCNHs to improve the ionic conductivity of the ionic liquid and to develop highly efficient electrolytes for ssDSSCs - Figure 4. [EMIM]I / SWCNHs mixtures of 10:1, 5:1, and $1: 1 \mathrm{wt} \%$ were prepared. For quasi-solid state electrolytes and a better pore penetration, the crystal growth inhibitor 1-butyl3-methylimidazolium tetrafuoroborate ([BMIM] $\left.\left[\mathrm{BF}_{4}\right]\right)$, 4-tert-butyl pyridine (TBP), and guanidinium thiocyanate (GuSCN) were added. Upon SWCNH-addition, $D_{\text {diff }}$ in symmetric cells gradually increased from 0.08 to $5.6 \times 10^{-10} \mathrm{~m}^{2} \mathrm{~s}^{-1}$ for pure [EMIM]I (E1) and $1: 1 \mathrm{wt} \%$ [EMIM]I / SWCNH (E2), respectively. The same trend was observed in quasi-solid state electrolytes without (E3) and with (E4) SWCNHs. Here, the corresponding $D_{\text {diff }} \mathrm{s}$ improved from 0.3 to $6.4 \times 10^{-10}$ $\mathrm{m}^{2} \mathrm{~s}^{-1}$. Furthermore, a gradual decrease of $R_{c t} \mathrm{~s}$ was observed upon SWCNH-addition indicating catalytic activity of SWCNHs inside the bulk electrolyte: $R_{c t}$ s decreased from 190.5 to $7.4 \Omega$ for symmetric cells with E1 and E2, and from 167.3 to 41.2 for $\mathbf{E 3}$ and E4.

In $\mathrm{TiO}_{2}$-based DSSCs sensitized with $\mathbf{N 7 1 9}$, a boost in $J_{s c} \mathrm{~s}$ was observed, when SWCNHs were present in the electrolyte: ssDSSCs with $\mathbf{E 2}$ featured $V_{o c} \mathrm{~s}$ of $0.45 \mathrm{~V}, J_{s c} \mathrm{~s}$ of $8.29 \mathrm{~mA} \mathrm{~cm}^{-2}, F F \mathrm{~s}$ of 0.56 , and, efficiencies of $2.1 \%$. On the contrary, ssDSSC with E1 showed $V_{o c} \mathrm{~s}$ of $0.47 \mathrm{~V}, J_{s c}$ of $1.27 \mathrm{~mA} \mathrm{~cm}{ }^{-2}, F F \mathrm{~s}$ of 0.71 , and efficiencies of $0.4 \%$. Mainly responsible for the 5-fold improvement in efficiencies was an intensification in $J_{s c} \mathrm{~s}$ upon addition of SWCNHs to the electrolyte. ssDSSCs with $\mathbf{E 3}$ exhibited $V_{o c}$ s of $0.74 \mathrm{~V}, J_{s c} \mathrm{~s}$ of $1.17 \mathrm{~mA}$ $\mathrm{cm}^{-2}, F F$ s of 0.70 , and efficiencies of $0.61 \%$, while ssDSSCs with E4 exhibited $V_{o c} \mathrm{~s}$ of $0.76 \mathrm{~V}, J_{s c} \mathrm{~s}$ of $16.03 \mathrm{~mA} \mathrm{~cm}{ }^{-2}, F F \mathrm{~s}$ of 0.64 , and efficiencies of $7.84 \%$. Notably, DSSCs with E3 and E4 featured comparable $V_{o c}$ s and $F F$ s. Only $J_{s c}$ s increased by a factor of nearly 
6, which is also valid, when comparing devices with E1 and E2. The increase in $V_{o c}$ s of ssDSSCs with $\mathbf{E 3}$ and $\mathbf{E 4}$ compared with those containing $\mathbf{E} 1$ and $\mathbf{E 2}$ is ascribed to the use of additives. ${ }^{75}$

As a complement, time-dependent J-V and EIS measurements were conducted to probe interactions between SWCNHs and the electrolyte. As time progresses, ssDSSCs with $\mathbf{E 3}$ and $\mathbf{E 4}$ featured nearly similar $V_{o c}$ s, but the $J_{s c}$ s decreased by nearly $75 \%$ for ssDSSCs with $\mathbf{E 3}$ after 5 days. In contrast, $J_{s c}$ s of devices with $\mathbf{E} 4$ showed a loss of only $9 \%$ during the same time period. This is likely related to the dye degradation via the substitution of the $\mathrm{SCN}^{-}$groups by $\mathrm{I}_{3}{ }^{-}$or polyiodides formed at the electrode surface. ${ }^{128,129}$ Thus, SWCNHs give rise to efficient electrolyte regeneration and prevent dye degradation. A gradual increase of $F F$ s in ssDSSCs with $\mathbf{E 4}$ was noted. Simultaneously, ssDSSCs with $\mathbf{E} 4$ exhibited a $71 \%$ increase in $D_{\text {diff }}$ s. The efficiencies dropped by $37 \%$ after 10 days for ssDSSCs with E3. In contrast, a rise by $22 \%$ during the same time period was noted, when $\mathbf{E} \mathbf{4}$ was implemented into DSSCs. From time-dependent Raman assays, an increase of the $\mathrm{I}_{\mathrm{D}} / \mathrm{I}_{\mathrm{G}}$ ratio of SWCNHs exposed to the electrolyte from 1.09 in a fresh device to 1.22 in 4-days aged electrolytes was observed. Afterwards, the $\mathrm{I}_{\mathrm{D}} / \mathrm{I}_{\mathrm{G}}$ ratio remained constant, which is perfectly in line with the increase of $D_{\text {diff }}$ s. In summary, the presence of SWCNHs in (quasi) solid-state electrolytes improved the ionic diffusivity and resulted in catalytically active material. Therefore, $R_{d i f f} \mathrm{~s}$ and $R_{c t} \mathrm{~s}$ were greatly reduced upon SWCNH-addition.

\section{Conclusions and Outlook}

At a glance, the impact of SWCNHs as i) integrative component into the semiconductor, ii) buffer layer, iii) replacement for platinum in the CE, and iv) additive for (quasi) solid-state electrolytes has been reviewed. Due to their good dispersibility, SWCNHs are easily applied by spin-coating, drop-casting, spray-coating, doctor-blading, etc. As such, the device performance and long-term stability were improved with SWCNHs present in each part of the DSSC. However, several major challenges need to be addressed in the future.

Firstly, electrical conductivity and transparency of SWCNHs limit their application as integrative component in $\mathrm{TiO}_{2}$ and as buffer layers. As a further step toward improving the device performance, SWCNHs could be tuned in terms of conductivity by chemical functionalization. Secondly, a detailed study of the active area combined with a control of the $\mathrm{I}_{\mathrm{D}} / \mathrm{I}_{\mathrm{G}}$ ratios without compromising the electrical conductivity of the SWCNH-based CEs is necessary to understand the underlying processes at the electrolyte|CE interface. Thirdly, their use as additives in solid-state electrolytes is limited by miscibility with ionic liquids. Therefore, the direct functionalization of SWCNHs with ionic liquids could be beneficial. Additionally, whether the electrolyte is directly regenerated by the SWCNHs in the bulk of the electrolyte before reaching the CE needs to be further investigated. To this end, a comprehensive study with focus on the impact of the type of defects or functionalization in SWCNHs on the electrolyte regeneration should be conducted. This also affects the SWCNH-based CE optimization.

Finally, the application of SWCNHs for highly efficient organicinorganic metal halide perovskite solar cells (PSC) should be addressed. So far, nanocarbons, such as fullerenes, SWCNTs, MWCNTs, and graphene, were tested as electron donors and acceptors in PSCs. ${ }^{130}$ For example, fullerene derivatives, such as PCBM, were used as hole transport layers and integrative component to reduce hysteresis and charge recombination. ${ }^{131-134}$ Furthermore, SWCNTs and MWCNTs were used as dopants for HTMs, such as Spiro-MeOTAD, ${ }^{135,136}$ as integrative component into the perovskite layer, ${ }^{137,138}$ and as hole extraction layer. ${ }^{139,140}$ As such, charge recombination losses were reduced and hole collection efficiencies were increased. Furthermore, the resistance toward water intrusion is enhanced upon applying a carbon nanotube-based layer enhancing the solar cell stability. ${ }^{139}$ rGO was incorporated into PEDOT:PSS to improve the hole transport properties. ${ }^{141}$ Given their similar properties, SWCNHs could be used as alternatives to the abovementioned nanocarbon applications for PSCs. This could further enhance their performance or long-term stability.
Taking all of the aforementioned into account, the use of SWCNHs in DSSCs has evolved into an active field, featuring notable breakthroughs. ${ }^{142-147}$ However, a full-fledged understanding on the underlying mechanisms is necessary to build the basis for the application on an industrial scale.

\section{Acknowledgments}

F. L., R. D. C., and D. M. G. acknowledge the EAM cluster in the frame of the DFG excellence programs for their support. D. M. G. acknowledges the DFG, ICMM, and the ZMP for financial and intellectual support.

\section{References}

1. S. Ito, T. N. Murakami, P. Comte, P. Liska, C. Grätzel, M. K. Nazeeruddin, and M. Grätzel, Thin Solid Films, 516, 4613 (2008).

2. J. E. Trancik, S. C. Barton, and J. Hone, Nano Lett., 8, 982 (2008).

3. S. K. Pathak, A. Abate, T. Leijtens, D. J. Hollman, J. Teuscher, L. Pazos, P. Docampo, U. Steiner, and H. J. Snaith, Adv. Energy Mater, 4, 1301667 (2014).

4. N.-G. Park, J. Van De Lagemaat, H. M. Cheong, A. Mascarenhas, and A. J. Frank, J. Phys. Chem. B, 103, 3308 (1999).

5. P. Cameron and L. Peter, J. Phys. Chem. B, 107, 14394 (2003).

6. C. H. Yoon, J. Lee, W.-S. Chae, and K.-J. Kim, Electrochim. Acta, 53, 2890 (2008)

7. B. Zhang, D. Wang, Y. Hou, S. Yang, X. H. Yang, J. H. Zhong, J. Liu, H. F. Wang, P. Hu, H. J. Zhao, and H. G. Yang, Sci. Rep., 3, 1836 (2013).

8. Z. Sun, M. Liang, and J. Chen, Acc. Chem. Res., 48, 1541 (2015).

9. A. Hauch and A. Georg, Electrochim. Acta, 46, 3457 (2001).

10. L. M. Gonçalves, V. de Zea Bermudez, H. A. Ribeiro, and A. M. Mendes, Energy Environ. Sci., 1, 655 (2008).

11. K. Sasaki, Y. Sekine, K. Tateno, and H. Gotoh, Phys. Rev. Lett., 111, 116801 (2013)

12. M. Yudasaka, S. Iijima, V. H. Crespi, A. Jorio, G. Dresselhaus, and M. S. Dresselhaus, Top. Appl. Phys., 111, 605 (2008).

13. D. Kasuya and M. Yudasaka, J. Phys. Chem. B, 106, 4947 (2002).

14. P. J. F. Harris, S. C. Tsang, J. B. Claridge, and M. L. H. Green, J. Chem. Soc. Faraday Trans., 90, 2799 (1994).

15. M. Yudasaka, S. Iijima, and V. Crespi, Top. Appl. Phys. Carbon Nanotub., 111, 605 (2008).

16. S. Zhu and G. Xu, Nanoscale, 2, 2538 (2010)

17. N. Karousis, I. Suarez-Martinez, C. P. Ewels, and N. Tagmatarchis, Chem. Rev. 116, 4850 (2016)

18. Z. Zhang, S. Han, C. Wang, J. Li, and G. Xu, Nanomaterials, 5, 1732 (2015)

19. S. Berber, Y.-K. Kwon, and D. Tománek, Phys. Rev. B, 62, R2291 (2000).

20. A. Javey, J. Guo, Q. Wang, M. Lundstrom, and H. Dai, Nature, 424, 654 (2003).

21. R. D. Costa, F. Lodermeyer, R. Casillas, and D. M. Guldi, Energy Environ. Sci., 7, 1281 (2014).

22. R. Casillas, F. Lodermeyer, R. D. Costa, M. Prato, and D. M. Guldi, Adv. Energy Mater, 4, 1301577 (2014)

23. F. Lodermeyer, M. Prato, R. D. Costa, and D. M. Guldi, Nanoscale, 8, 7556 (2016)

24. T. Azami, D. Kasuya, R. Yuge, M. Yudasaka, S. Iijima, T. Yoshitake, and Y. Kubo, J. Phys. Chem. C, 112, 1330 (2008).

25. R. Yuge, S. Bandow, K. Nakahara, M. Yudasaka, K. Toyama, T. Yamaguchi, S. Iijima, and T. Manako, Carbon, 75, 322 (2014).

26. T. Yamaguchi, S. Bandow, and S. Iijima, Chem. Phys. Lett., 389, 181 (2004).

27. H. Takikawa, M. Ikeda, K. Hirahara, Y. Hibi, Y. Tao, P. A. Ruiz, T. Sakakibara, S. Itoh, and S. Iijima, Phys. B Condens. Matter, 323, 277 (2002).

28. M. Ikeda, H. Takikawa, T. Tahara, Y. Fujimura, M. Kato, K. Tanaka, S. Itoh, and T. Sakakibara, Japanese J. Appl. Physics, Part 2 Lett., 41, 852 (2002).

29. S. Bandow, A. M. Rao, G. U. Sumanasekera, P. C. Eklund, F. Kokai, K. Takahashi, M. Yudasaka, and S. Iijima, Appl. Phys. A Mater. Sci. Process., 71, 561 (2000).

30. H. M. Heise, R. Kuckuk, A. Srivastava, and B. P. Asthana, J. Raman Spectrosc., 42 294 (2011)

31. F. Tuinstra and J. L. Koenig, J. Chem. Phys., 53, 1126 (1970).

32. F. Lodermeyer, R. D. Costa, R. Casillas, F. T. U. Kohler, P. Wasserscheid, M. Prato, and D. M. Guldi, Energy Environ. Sci., 8, 241 (2015).

33. D. V. Kolesnikov and V. A. Osipov, J. Exp. Theor. Phys. Lett., 79, 532 (2004).

34. K. Urita, S. Seki, S. Utsumi, D. Noguchi, H. Kanoh, H. Tanaka, Y. Hattori, Y. Ochiai, N. Aoki, M. Yudasaka, S. Iijima, and K. Kaneko, Nano Lett., 6, 1325 (2006).

35. K. Urita, S. Seki, H. Tsuchiya, H. Honda, S. Utsumi, C. Hayakawa, H. Kanoh, T. Ohba, H. Tanaka, M. Yudasaka, S. Iijima, and K. Kaneko, J. Phys. Chem. C, 112, 8759 (2008)

36. C.-M. Yang, D. Kasuya, M. Yudasaka, S. Iijima, and K. Kaneko, J. Phys. Chem. B, 108, 17775 (2004)

37. S. M. Unni, S. N. Bhange, R. Illathvalappil, N. Mutneja, K. R. Patil, and S. Kurungot, Small, 11, 352 (2015)

38. S. Bandow, F. Kokai, K. Takahashi, M. Yudasaka, and S. Iijima, Appl. Phys. A Mater. Sci. Process., 73, 281 (2001).

39. H. Imai, P. K. Babu, E. Oldfield, A. Wieckowski, D. Kasuya, T. Azami, Y. Shimakawa, M. Yudasaka, Y. Kubo, and S. Iijima, Phys. Rev. B, 73, 125405 (2006).

40. J.-M. Bonard, R. Gaál, S. Garaj, L. Thien-Nga, L. Forró, K. Takahashi, F. Kokai, M. Yudasaka, and S. Iijima, J. Appl. Phys., 91, 10107 (2002).

41. X. Fan, J. Tan, G. Zhang, and F. Zhang, Nanotechnology, 18, 195103 (2007) 
42. K. Ajima, M. Yudasaka, T. Murakami, A. Maigne, K. Shiba, and S. Iijima, Mol. Pharm., 2, 475 (2005).

43. K. Ajima, T. Murakami, Y. Mizoguchi, K. Tsuchida, T. Ichihashi, S. Iijima, and M. Yudasaka, ACS Nano, 2, 2057 (2008).

44. T. Murakami, H. Sawada, G. Tamura, M. Yudasaka, S. Iijima, and K. Tsuchida, Nanomedicine, 3, 453 (2008).

45. S. Matsumura, S. Sato, M. Yudasaka, A. Tomida, T. Tsuruo, S. Iijima, and K. Shiba, Mol. Pharm., 6, 441 (2009).

46. J. Miyawaki, S. Matsumura, R. Yuge, T. Murakami, S. Sato, A. Tomida, T. Tsuruo, T. Ichihashi, T. Fujinami, H. Irie, K. Tsuchida, S. Iijima, K. Shiba, and M. Yudasaka, ACS Nano, 3, 1399 (2009).

47. A. J. Zambano, S. Talapatra, K. Lafdi, M. T. Aziz, W. McMillin, G. Shaughnessy, A. D. Migone, M. Yudasaka, S. Iijima, F. Kokai, and K. Takahashi, Nanotechnology, 13, 201 (2002)

48. N. H. Quang, M. Van Trinh, B. Lee, and J. Huh, Sensors Actuators B Chem., 113, 341 (2006).

49. J. Fan and M. Yudasaka, Chem. Phys. Lett., 397, 5 (2004).

50. T. Ohkubo, Y. Hattori, H. Kanoh, T. Konishi, H. Sakai, M. Abe, D. Kasuya M. Yudasaka, and S. Iijima, Phys. Scr., T115, 685 (2005).

51. N. Sano and S. Ukita, Mater Chem. Phys., 99, 447 (2006).

52. S. M. Unni, R. Illathvalappil, S. N. Bhange, H. Puthenpediakkal, and S. Kurungot, ACS Appl. Mater. Interfaces, 7, 24256 (2015).

53. S. M. Unni, S. Ramadas, R. Illathvalappil, S. N. Bhange, and S. Kurungot, J. Mater Chem. A, 3, 4361 (2015).

54. D. Wen, L. Deng, M. Zhou, S. Guo, L. Shang, G. Xu, and S. Dong, Biosens. Bioelectron., 25, 1544 (2010).

55. D. Wen, X. Xu, and S. Dong, Energy Environ. Sci., 4, 1358 (2011).

56. K. Momeni and R. S. Yassar, J. Comput. Theor. Nanosci., 7, 1035 (2010).

57. A. F. S. Blazewicz, J. Mater. Sci., 46, 5680 (2011).

58. N. W. Duffy, L. M. Peter, R. M. G. Rajapakse, and K. G. U. Wijayantha, Electrochem. Commun., 2, 658 (2000).

59. M. Adachi, M. Sakamoto, J. Jiu, Y. Ogata, and S. Isoda, J. Phys. Chem. B, 110, $13872(2006)$

60. A. Listorti, B. O'Regan, and J. R. Durrant, Chem. Mater, 23, 3381 (2011)

61. M. Batmunkh, M. J. Biggs, and J. G. Shapter, Adv. Sci., 2, 2198 (2015).

62. H. Kim and G. Moon, J. Phys. Chem. C, 116, 1535 (2011).

63. C.-Y. Yen, Y.-F. Lin, S.-H. Liao, C.-C. Weng, C.-C. Huang, Y.-H. Hsiao, C.-C. M. Ma, M.-C. Chang, H. Shao, M.-C. Tsai, C.-K. Hsieh, C.-H. Tsai, and F.-B. Weng, Nanotechnology, 19, 375305 (2008).

64. D. M. Guldi and R. D. Costa, J. Phys. Chem. Lett., 4, 1489 (2013).

65. R. D. Costa, S. Feihl, A. Kahnt, S. Gambhir, D. L. Officer, G. G. Wallace, M. I. Lucio, M. A. Herrero, E. Vázquez, Z. Syrgiannis, M. Prato, and D. M. Guldi, Adv. Mater, 25, 6513 (2013).

66. S. L. Kim, S.-R. Jang, R. Vittal, J. Lee, and K.-J. Kim, J. Appl. Electrochem., 36, 1433 (2006).

67. Z. Niu, L. Liu, L. Zhang, W. Zhou, X. Chen, and S. Xie, Adv. Energy Mater, 5, 1500677 (2015).

68. G. Lu, K. Yu, Z. Wen, and J. Chen, Nanoscale, 5, 1353 (2013).

69. D. Benetti, K. T. Dembele, J. Benavides, H. Zhao, S. Cloutier, I. Concina, A. Vomiero, and F. Rosei, J. Mater. Chem. C, 4, 3555 (2016).

70. N. Yang, J. Zhai, D. Wang, Y. Chen, and L. Jiang, ACS Nano, 4, 887 (2010).

71. S. Sun, L. Gao, and Y. Liu, Appl. Phys. Lett., 96, 083113 (2010).

72. J. Halme, P. Vahermaa, K. Miettunen, and P. Lund, Adv. Mater, 22, E210 (2010).

73. K. Zhu, S. Jang, and A. Frank, J. Phys. Chem. Lett., 2, 1070 (2011).

74. L. Peter, Chem. Rev., 90, 753 (1990).

75. M. K. Nazeeruddin, A. Kay, E. Müller, P. Liska, N. Vlachopoulos, and M. Grätzel, J. Am. Chem. Soc., 115, 6382 (1993).

76. T. He, Y. F. Wang, and J. H. Zeng, J. Mater. Chem. C, 4, 8235 (2016)

77. A. K. K. Kyaw, H. Tantang, T. Wu, L. Ke, C. Peh, Z. H. Huang, X. T. Zeng, H. V. Demir, Q. Zhang, and X. W. Sun, Appl. Phys. Lett., 99, 18 (2011)

78. Y. Tang, C. Lee, J. Xu, Z. Liu, and Z. Chen, ACS Nano, 4, 3482 (2010).

79. L. Kavan and M. Grätzel, Electrochim. Acta, 40, 643 (1995).

80. A. Zaban, M. Greenshtein, and J. Bisquert, ChemPhysChem, 4, 859 (2003).

81. J. Halme, Phys. Chem. Chem. Phys., 13, 12435 (2011).

82. G. Schlichthörl, S. Y. Huang, J. Sprague, and A. J. Frank, J. Phys. Chem. B, 101, 8141 (1997).

83. S. R. Kim, M. K. Parvez, and M. Chhowalla, Chem. Phys. Lett., 483, 124 (2009).

84. A. Yella, H.-W. Lee, H. N. Tsao, C. Yi, A. K. Chandiran, M. K. Nazeeruddin, E. W.-G. Diau, C.-Y. Yeh, S. M. Zakeeruddin, and M. Grätzel, Science, 334, 629 (2011).

85. G. Lozano, S. Colodrero, O. Caulier, M. E. Calvo, and H. Míguez, J. Phys. Chem. $C, \mathbf{1 1 4}, 3681$ (2010).

86. G. Boschloo and A. Hagfeldt, Acc. Chem. Res., 42, 1819 (2009).

87. G. Yue, J. Wu, Y. Xiao, M. Huang, J. Lin, and J.-Y. Lin, J. Mater. Chem. A, 1, 1495 (2013).

88. C.-L. Wang, J.-Y. Liao, S.-H. Chung, and A. Manthiram, Adv. Energy Mater, 5, 1401524 (2014).

89. S. Shukla, N. H. Loc, P. P. Boix, T. M. Koh, and R. R. Prabhakar, ACS Nano, 8, 10597 (2014)

90. S. R. Taylor, Geochim. Cosmochim. Acta, 28, 1273 (1964).

91. M. Rehkamper, A. N. Halliday, D. Barfod, and J. G. Fitton, Science, 278, 1595 (1997).

92. S. Yun, A. Hagfeldt, and T. Ma, Adv. Mater., 26, 6210 (2014).

93. Q. W. Jiang, G. R. Li, and X. P. Gao, Chem. Commun., 6720 (2009).

94. H. Zhou, Y. Shi, L. Wang, H. Zhang, C. Zhao, A. Hagfeldt, and T. Ma, Chem. Commun., 49, 7626 (2013).
95. Q. Tai and X. Zhao, J. Mater Chem. A, 2, 13207 (2014).

96. H. Wang, Q. Feng, F. Gong, Y. Li, and G. Zhou, J. Mater. Chem. A, 1, 97 (2013).

97. T. N. Murakami, S. Ito, Q. Wang, M. K. Nazeeruddin, T. Bessho, I. Cesar, P. Liska, R. Humphry-Baker, P. Comte, P. Péchy, and M. Graätzel, J. Electrochem. Soc., 153, A2255 (2006).

98. X. Meng, C. Yu, X. Song, Y. Liu, S. Liang, Z. Liu, C. Hao, and J. Qiu, Adv. Energy Mater, 5, 1500180 (2015)

99. H. Wang and Y. H. Hu, Energy Environ. Sci., 5, 8182 (2012).

100. J. G. Nam, Y. J. Park, B. S. Kim, and J. S. Lee, Scr. Mater, 62, 148 (2010).

101. L. Kavan, J.-H. Yum, and M. Grätzel, Nano Lett., 11, 5501 (2011).

102. L. Kavan, J.-H. Yum, M. Nazeeruddin, and Mohammad Khaja Grätzel, ACS Nano, 5, $9171(2011)$

103. S. Huang, Z. Yang, L. Zhang, R. He, T. Chen, Z. Cai, Y. Luo, H. Lin, H. Cao, X. Zhu, and H. Peng, J. Mater. Chem., 22, 16833 (2012).

104. B. Lee, D. B. Buchholz, and R. P. H. Chang, Energy Environ. Sci., 5, 6941 (2012).

105. M. Adachi, K. Noda, R. Tanino, J. Adachi, K. Tsuchiya, Y. Mori, and F. Uchida, Chem. Lett., 40, 890 (2011).

106. K. Susmitha, M. M. Kumari, A. J. Berkmans, M. N. Kumar, L. Giribabu, S. V. Manorama, and M. Raghavender, Sol. Energy, 133, 524 (2016).

107. W. Kwon, J. Kim, and S. Rhee, J. Mater. Chem. A, 1, 3202 (2013).

108. S. M. Feldt, P. W. Lohse, F. Kessler, M. K. Nazeeruddin, M. Grätzel, G. Boschloo, and A. Hagfeldt, Phys. Chem. Chem. Phys., 15, 7087 (2013).

109. T. Stergiopoulos and P. Falaras, Adv. Energy Mater., 2, 616 (2012)

110. J. Liu, S. K. Pathak, T. Stergiopoulos, T. Leijtens, K. Wojciechowski, S. Schumann, N. Kausch-Busies, and H. J. Snaith, J. Phys. Chem. Lett., 6, 1666 (2015)

111. D. Pysch, A. Mette, and S. W. Glunz, Sol. Energy Mater. Sol. Cells, 91, 1698 (2007).

112. C. Law, S. C. Pathirana, X. Li, A. Y. Anderson, P. R. F. Barnes, A. Listorti, T. H. Ghaddar, and B. C. O'Regan, Adv. Mater, 22, 4505 (2010).

113. C. Law, O. Moudam, S. Villarroya-Lidon, and B. O'Regan, J. Mater. Chem., 22, 23387 (2012).

114. J. Kim, J. K. Koh, B. Kim, S. H. Ahn, H. Ahn, D. Y. Ryu, J. H. Kim, and E. Kim, Adv. Funct. Mater, 21, 4633 (2011).

115. S. H. Ahn, W. S. Chi, D. J. Kim, S. Y. Heo, and J. H. Kim, Adv. Funct. Mater, 23, 3901 (2013)

116. S. H. Ahn, W. S. Chi, J. T. Park, J. K. Koh, D. K. Roh, and J. H. Kim, Adv. Mater. 24, 519 (2012).

117. S. H. Ahn, J. H. Koh, J. A. Seo, and J. H. Kim, Chem. Commun., 46, 1935 (2010).

118. J. Burschka, F. Kessler, M. K. Nazeeruddin, and M. Grätzel, Chem. Mater., 25, 2986 (2013).

119. B. Xu, E. Gabrielsson, M. Safdari, M. Cheng, Y. Hua, H. Tian, J. M. Gardner, L. Kloo, and L. Sun, Adv. Energy Mater, 5, 1402340 (2015).

120. M. Freitag, Q. Daniel, M. Pazoki, K. Sveinbjörnsson, J. Zhang, L. Sun, A. Hagfeldt, and G. Boschloo, Energy Environ. Sci., 8, 2634 (2015).

121. C.-P. Lee, P.-Y. Chen, R. Vittal, and K.-C. Ho, J. Mater. Chem., 20, 2356 (2010).

122. B.-X. Lei, W.-J. Fang, Y.-F. Hou, J.-Y. Liao, D.-B. Kuang, and C.-Y. Su, J. Photochem. Photobiol. A Chem., 216, 8 (2010).

123. Y.-H. Chang, P.-Y. Lin, S.-R. Huang, K.-Y. Liu, and K.-F. Lin, J. Mater. Chem., 22, 15592 (2012)

124. C.-P. Lee, L.-Y. Lin, P.-Y. Chen, R. Vittal, and K.-C. Ho, J. Mater. Chem., 20, 3619 (2010).

125. M. S. Akhtar, S. Kwon, F. J. Stadler, and O. B. Yang, Nanoscale, 5, 5403 (2013).

126. L. J. Brennan, M. T. Byrne, M. Bari, and Y. K. Gun'ko, Adv. Energy Mater, 1, 472 (2011).

127. V. M. Mohan, K. Murakami, A. Kono, and M. Shimomura, J. Mater. Chem. A, 1, 7399 (2013).

128. H. Greijer, J. Lindgren, and A. Hagfeldt, J. Phys. Chem. B, 105, 6314 (2001).

129. J. R. Jennings, Y. Liu, and Q. Wang, J. Phys. Chem. C, 115, 15109 (2011).

130. S. Fukuzumi, ECS J. Solid State Sci. Technol., 6, M3055 (2017)

131. J. Xu, A. Buin, A. H. Ip, W. Li, O. Voznyy, R. Comin, M. Yuan, S. Jeon, Z. Ning, J. J. McDowell, P. Kanjanaboos, J.-P. Sun, X. Lan, L. N. Quan, D. H. Kim, I. G. Hill, P. Maksymovych, and E. H. Sargent, Nat. Commun., 6, 7081 (2015).

132. Z. Zhu, Q. Xue, H. He, K. Jiang, Z. Hu, Y. Bai, T. Zhang, S. Xiao, K. Gundogdu, B. R. Gautam, H. Ade, F. Huang, K. S. Wong, H.-L. Yip, S. Yang, and H. Yan, $A d v$. Sci., 3, 1500353 (2016).

133. M. Valles-Pelarda, B. C. Hames, I. García-Benito, O. Almora, A. Molina-Ontoria, R. S. Sánchez, G. Garcia-Belmonte, N. Martín, and I. Mora-Sero, J. Phys. Chem. Lett., 7, 4622 (2016).

134. J. Han, H.-Y. Wang, Y. Wang, M. Yu, S. Yuan, P. Sun, Y. Qin, Z.-X. Guo, J.-P. Zhang, and X.-C. Ai, RSC Adv., 6, 112512 (2016).

135. K. Aitola, K. Sveinbjörnsson, J.-P. Correa-Baena, A. Kaskela, A. Abate, Y. Tian, E. M. J. Johansson, M. Grätzel, E. I. Kauppinen, A. Hagfeldt, and G. Boschloo, Energy Environ. Sci., 9, 461 (2016).

136. T. Miletić, E. Pavoni, V. Trifiletti, A. Rizzo, A. Listorti, S. Colella, N. Armaroli, and D. Bonifazi, ACS Appl. Mater. Interfaces, 8, 27966 (2016).

137. P. Schulz, A.-M. Dowgiallo, M. Yang, K. Zhu, J. L. Blackburn, and J. J. Berry, J. Phys. Chem. Lett., 7, 418 (2016).

138. Y. Zhang, L. Tan, Q. Fu, L. Chen, T. Ji, X. Hu, and Y. Chen, Chem. Commun., 52, 5674 (2016) 
139. H. Li, K. Cao, J. Cui, S. Liu, X. Qiao, Y. Shen, and M. Wang, Nanoscale, 8, 6379 (2016).

140. Z. Wei, H. Chen, K. Yan, X. Zheng, and S. Yang, J. Mater. Chem. A, 3, 24226 (2015).

141. X. Huang, H. Guo, J. Yang, K. Wang, X. Niu, and X. Liu, Org. Electron., 39, 288 (2016).

142. D. M. Guldi and R. D. Costa, J. Phys. Chem. Lett., 4, 1489 (2013).
143. J. T. Margraf, F. Lodermeyer, V. Strauss, P. Haines, J. Walter, W. Peukert, R. D. Costa, T. Clark, and D. M. Guldi, Nanoscale Horiz., 1, 220 (2016).

144. F. Lodermeyer, R. D. Costa, and D. M. Guldi, Adv. Energy Mater, 1601883 (2017).

145. D. M. Guldi and V. Sgobba, Chem. Commun., 47, 606 (2011).

146. M. Rudolf, S. V. Kirner, and D. M. Guldi, Chem. Soc. Rev., 45, 612 (2016).

147. V. Strauss, A. Roth, M. Sekita, and D. Guldi, Chem, 1, 531 (2016). 